\title{
Developments of Two High-speed Dual-channel VCSEL Driver ASICs
}

\section{Wei Zhou ${ }^{1}$}

Department of Physics, Central China Normal University, Department of Physics, Southern Methodist University

Wuhan, Hubei 430079, P.R. China

E-mail: weizhouesmu.edu

\section{Xiangming Sun ${ }^{2}$, Le Xiao, Guangming Huang}

Department of Physics, Central China Normal University

Wuhan, Hubei 430079, P.R. China

E-mail: xmsundphy.ccnu.edu.cn; lexiaolmails.ccnu.edu.cn;

gmhuangephy.ccnu.edu.cn

\section{Di Guo, Quan Sun, Datao Gong, Chonghan Liu, Tiankuan Liu, Jingbo Ye}

Department of Physics, Southern Methodist University

Dallas, Texas 75275, USA

E-mail: digesmu. edu; quansesmu.edu; dgongesmu. edu;kentI asmu.edu; tliuesmu. edu; yejbesmu. edu

\begin{abstract}
We present two designs of a dual-channel VCSEL driver ASIC, LOCld130 and LOCld65, for detector front-end readout via optical links. Each channel of the driver is designed to operate up to 5.12 Gbps or 14 Gbps respectively. They are implemented in commercial $130-\mathrm{nm}$ and $65-\mathrm{nm}$ CMOS technologies. Techniques that are adopted to extend the bandwidth are multiple stages, shared inductive peaking, active feedback and passive R-C. In the typical case the 5.12-Gbps driver dissipates $56 \mathrm{~mW} /$ channel (VCSEL included) and the 14-Gbps $58 \mathrm{~mW} /$ channel. LOCld65 will be tested in November 2017 and LOCld130 will be submitted for fabrication in the spring of 2018 .
\end{abstract}

Topical Workshop on Electronics for Particle Physics

11 - 14 September 2017

Santa Cruz, California

\footnotetext{
${ }^{1}$ Speaker

${ }^{2}$ Corresponding Author
} 


\section{Introduction}

Optical link, a high speed data transmission system has been extensively used in readout systems of high energy physics experiments for decades, benefiting from its high bandwidth and low power consumption. Based on years of R\&D, we have designed the dual-channel VCSEL driver, LOCld [1] in a commercial 250-nm Silicon-on-Sapphire CMOS technology as the baseline choice for the ATLAS Liquid-Argon-Calorimeter trigger upgrade [2], as shown in Figure 1. We design LOCld130 as a drop-in backup to LOCld in a commercial-130 nm CMOS technology. We also develop LOCld65 using the same 65-nm technology with which lpGBT [3] is being developed. lpGBT is the serializer-deserializer (SerDes) ASIC developed chiefly for detector upgrades for the HL-LHC. The serializer in lpGBT operates at 5.12 or $10.48 \mathrm{Gbps}$ and the deserializer at $2.56 \mathrm{Gbps}$. While the predecessor of lpGBT, the GBTx SerDes has a matching optical transceiver (VTRx) developed through the Versatile Link (VL) common project, the optical module being investigated in the VL+ common project will be based on array optics with an array VCSEL driver that has a different driving mechanism and is not suitable for single channel transceivers. Both LOCld130 and LOCld65 are dual-channel VCSEL drivers, but each channel in the ASIC is individually powered, making them suitable for applications in dualchannel optical transmitters such as MTx and VTTx or in transceivers such as MTRx and VTRx [4]. LOCld65 is to provide a perfect match with to lpGBT when the application does not call for array optics, and to benefit from the current development of optical modules of MTx and VTTx.

On-detector, rad-to

Off-detector, COTS

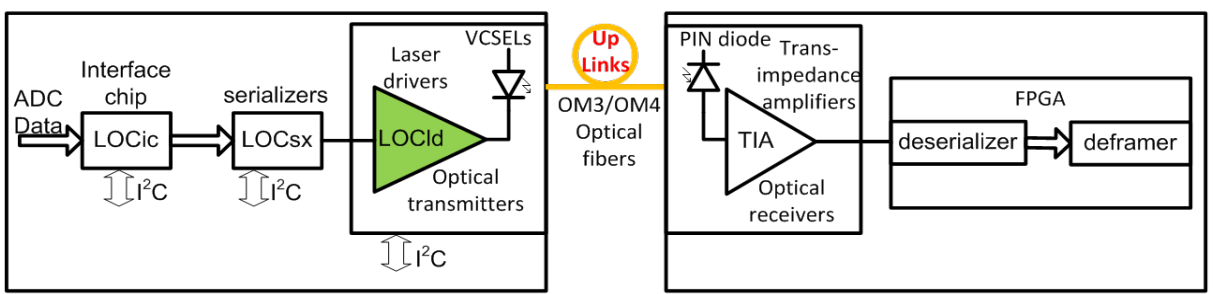

Figure 1: Optical link for the ATLAS liquid Argon calorimeter upgrade.

\section{Designs}

\subsection{LOCld130}

Although LOCld130 is a drop-in backup of LOCld, it has a simpler channel structure, lower power consumption, and fewer external interfaces. The analog core of LOCld130 has two parts: a two-stage limiting amplifier (LA) and a high-current differential driver. The minimum input signal to LOCld130 is $200 \mathrm{mV}_{\mathrm{P}-\mathrm{P}}$ and the data rate is up to $5.12 \mathrm{Gbps}$. The power supply for LOCld130 is $1.5 \mathrm{~V}$. We design the output signal of the LA with a swing of $1 \mathrm{~V}_{\mathrm{P}-\mathrm{P}}, 2 / 3$ of the power supply voltage. This way the LA will fully steer the last stage bias current from one arm of the differential pair to the other one to maximize electro-optical efficiency. In the LA design shown in Figure 2, we choose a two-stage amplifier to optimize the gain as well as the bandwidth, and a 3.2-nH peaking inductor shared between the two stages, boosting the bandwidth of LA more than $3.5 \mathrm{GHz}$ [5]. The bias current of each stage is $1.5 \mathrm{~mA}$ and $3 \mathrm{~mA}$ respectively. In the post-layout simulation, we use 1.5-nH inductors and a VCSEL equivalent 
model [6] to simulate the bonding wires inductance and VCSEL. An eye diagram of the current to the laser, through the whole channel post-layout simulation with PRBS7 input, is shown in Figure 3.

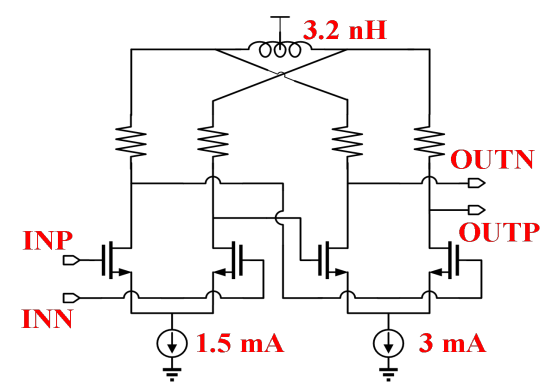

Figure 2: The schematic of the LA for LOCld130.

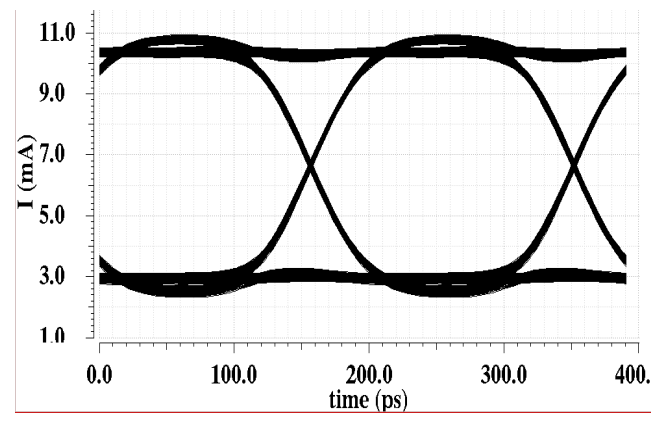

Figure 3: Typical eye diagram of LOCld130 at 5.12 Gpbs.

\subsection{LOCId65}

We also employ a full differential structure in LOCld65, similar to the design of LOCld130, as depicted in Figure 4. However LOCld65 is required to work under more rigorous conditions. The input signal is assumed to be $100 \mathrm{mV}_{\mathrm{P}-\mathrm{P}}$, and the data rate is $14 \mathrm{Gbps}$. In the design we also consider the high frequency loss in the input channel (PCB trace, bonding wire and the ESD diode). Shown in Figure 5(a), we design the first stage of the amplifier to be a continuous-time equalizer (CTLE) [7] to compensate the potential high-frequency signal loss. The peaking strength and the DC gain can be adjusted by tuning the resistor, depicted in Figure 6(a). We employ a four-stage LA as pre-driver shown in figure 5(b) [5]. Each of two successive gain cells shares one inductor to improve the bandwidth and to reduce chip area. The gain and bandwidth of the four-stage amplifier significantly depend on the process variation. For example, in the fast corner, the bandwidth of the amplifier is higher but the gain is smaller than typical case. To keep balance between the bandwidth and gain for all the process corner, we use an active-feedback-cell between the stages to adjust the gain and the bandwidth. By programming the feedback tail current, the total gain of the limiting amplifier is always greater than $18 \mathrm{~dB}$ and the bandwidth is higher than $10 \mathrm{GHz}$, depicted in Figure 6(b). The schematic of the output stage is shown in Figure 5(c). The output stage also employs the CTLE technique to compensate for channel loss. The amplifier's bias current is programmable with $\mathrm{I}^{2} \mathrm{C}$, offering a modulation current from $2 \mathrm{~mA}$ to $8 \mathrm{~mA}$ for the laser diode. Shown in Figure 7 is the eye diagram of the laser current, post-layout simulation with PRBS7 input. 


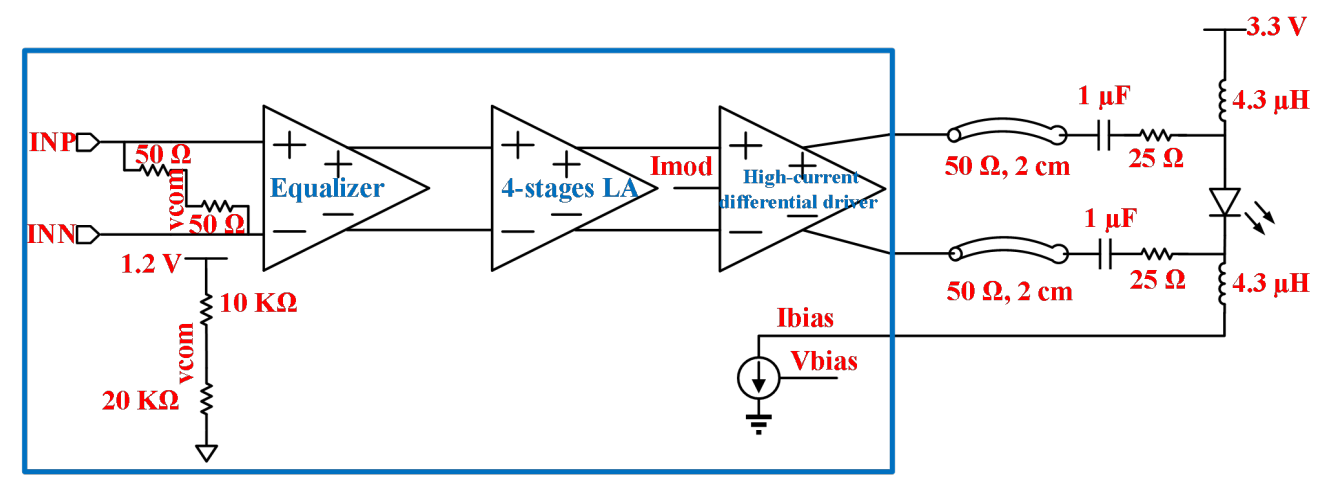

Figure 4: Block diagram of LOCld65 channel.

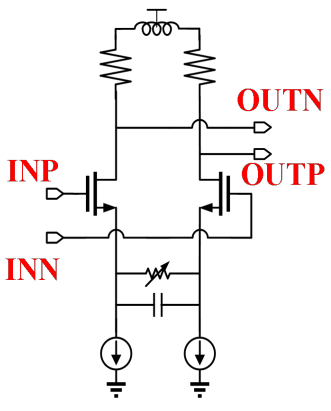

(a) CTLE

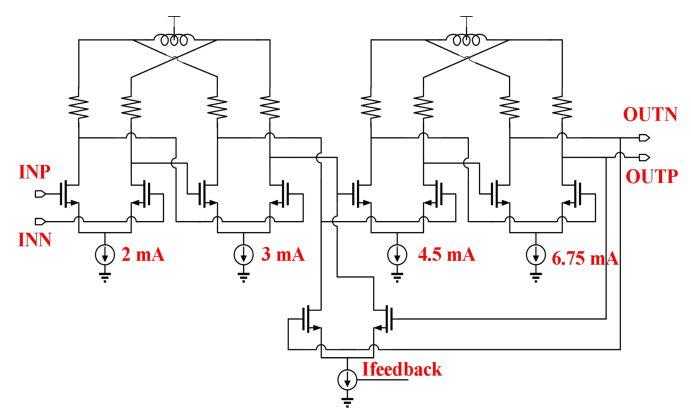

(b) Four-stage LA

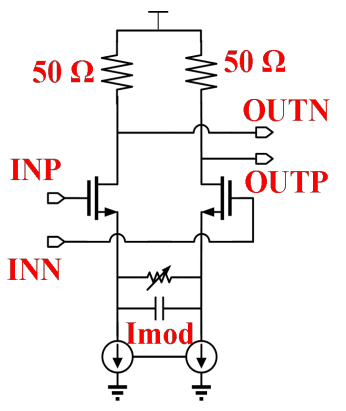

(c) Output stage

Figure 5: The schematic of each part in LOCld65 channel.

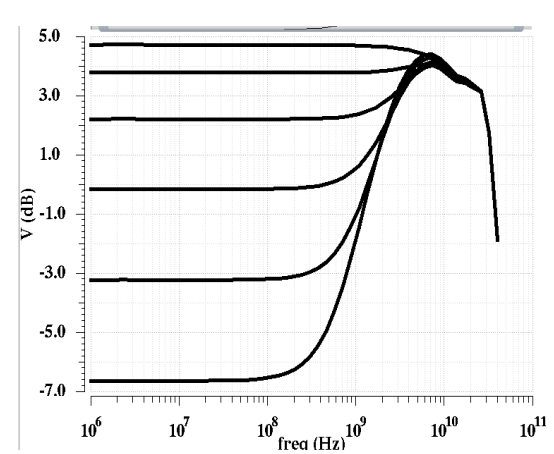

(a)

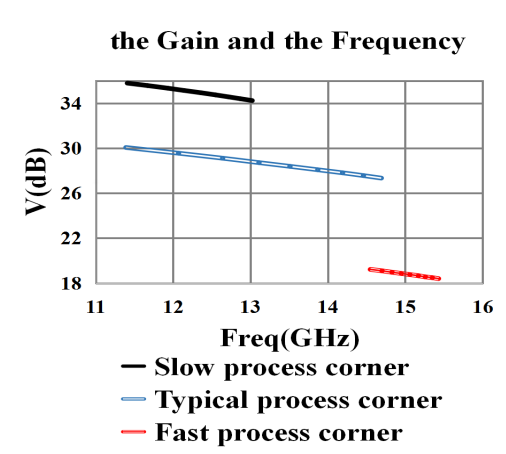

(b)

Figure 6: Simulated amplitude-frequency response curve of CTLE and four-stage LA.

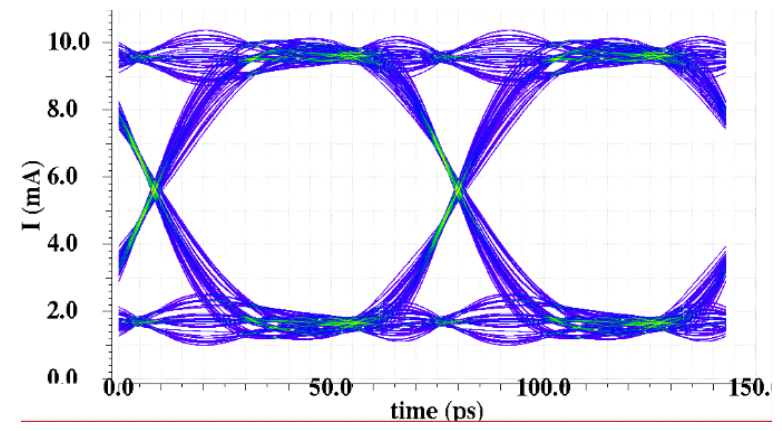

Figure 7: Typical eye diagram of LOCld65 at 14 Gpbs. 


\subsection{Conclusion}

We designed two high-speed Dual-channel VCSEL Driver ASICs, LOCld130 and LOCld65, using commercial $130-\mathrm{nm}$ and $65-\mathrm{nm}$ CMOS processes. LOCld130 is a $5.12 \mathrm{Gbps}$ VCSEL driver ASIC designed as a drop-in backup to LOCld. The total power consumption of LOCld130 is $112 \mathrm{~mW}$ (56 mW/channel) including the VCSELs. LOCld65 runs at 14 Gbps with a power consumption of $116 \mathrm{~mW}$ (58 mW/channel), VCSEL included. LOCld65 was submitted for fabrication in May 20th 2017. We expect to start the evaluation of this ASIC in November 2017. We also plan to submit LOCld130 for fabrication in the spring of 2018.

\section{Acknowledgments}

This work is supported by SMU's Dedman College Dean's Research Council Grant, the US Department of Energy Grant DE-FG02-04ER1299 and by the China Scholarship Council (CSC). The authors are grateful to Paulo Moreira, Syzmon Kulis and Sandro Bonacini from CERN for the $\mathrm{I}^{2} \mathrm{C}$ slave design.

\section{References}

[1] X. Li et al, 8-Gbps-per-channel radiation-tolerant VCSEL drivers for the LHC detector upgrade [J]. Journal of Instrumentation, 2015, 10(02): C02017.

[2] ATLAS collaboration, ATLAS liquid argon calorimeter Phase-I upgrade technical design report, CERN-LHCC-2013-017, ATLAS-TDR-022 (2013).

[3] P. Rodrigues et al., The LpGBT Project Status and Overview, ACES 2016 - Fifth Common ATLAS CMS Electronics Workshop for LHC Upgrades (2016).

[4] C. Liu et al., The Miniature Optical Transmitter and Transceiver for the High-Luminosity LHC (HLLHC) experiments, 2013 JINST 8 C12027.

[5] T. Huang, et al., A 28Gb/s 1pJ/b shared-inductor optical receiver with $56 \%$ chip-area reduction in $28 \mathrm{~nm}$ CMOS, ISSCC Dig. Tech. Papers, pp. 144-145, Feb. 2014.

[6] C. Ji, et al., High volume 850nm oxide VCSEL development for high bandwidth optical data link application, Proc. SPIE 7229, 722904 (2009).

[7] S. Gondi and B, Razavi, Equalization and clock and data recovery techniques for 10-Gb/s CMOS serial-link receivers, IEEE J. SolidState Circuits, vol. 42, no. 9, pp. 1999-2011, Sep. 2007. 